\title{
Pharmacological manipulation of arachidonic acid-epoxygenase results in divergent effects on renal damage
}

\author{
Jing Li ${ }^{1}$, Charles T. Stier ${ }^{1}$, Praveen N. Chander ${ }^{2}$, Vijay L. Manthati ${ }^{3}$, John R. Falck ${ }^{3}$ and \\ Mairéad A. Carroll ${ }^{1}$ *
}

' Department of Pharmacology, New York Medical College, Valhalla, NY, USA

${ }^{2}$ Department of Pathology, New York Medical College, Valhalla, NY, USA

${ }^{3}$ Department of Biochemistry, University of Texas Southwestern Medical Center, Dallas, TX, USA

\section{Edited by:}

John M. Seubert, University of

Alberta, Canada

\section{Reviewed by:}

John D. Imig, Medical College of Wisconsin, USA

Paul Jurasz, University of Alberta, Canada

\section{${ }^{*}$ Correspondence:}

Mairéad A. Carroll, Department of Pharmacology, New York Medical College, Room 541, Basic Science Building, 15 Dana Road, Valhalla, NY 10595, USA

e-mail: mairead_carroll@nymc.edu
Kidney damage is markedly accelerated by high-salt (HS) intake in stroke-prone spontaneously hypertensive rats (SHRSP). Epoxyeicosatrienoic acids (EETs) are epoxygenase products of arachidonic acid which possess vasodepressor, natriuretic, and anti-inflammatory activities. We examined whether up-regulation (clofibrate) or inhibition [N-methylsulfonyl-6-(2-propargyloxyphenyl)hexanamide (MS-PPOH)] of epoxygenase would alter systolic blood pressure (SBP) and/or renal pathology in SHRSP on HS intake $(1 \% \mathrm{NaCl}$ drinking solution). Three weeks of treatment with clofibrate induced renal cortical protein expression of CYP2C23 and increased urinary excretion of EETs compared with vehicle-treated SHRSP. SBP and urinary protein excretion (UPE) were significantly lowered with clofibrate treatment. Kidneys from vehicle-treated SHRSP, which were on HS intake for 3 weeks, demonstrated focal lesions of vascular fibrinoid degeneration, which were markedly attenuated with clofibrate treatment. In contrast, 2 weeks of treatment with the selective epoxygenase inhibitor, MS-PPOH, increased UPE without significantly altering neither urinary EET levels nor SBP. Kidneys from vehicle-treated SHRSP, which were on HS intake for 11 days, demonstrated occasional mild damage whereas kidneys from MS-PPOH-treated rats exhibited widespread malignant nephrosclerosis. These results suggest that pharmacological manipulation of epoxygenase results in divergent effects on renal damage and that interventions to increase EET levels may provide therapeutic strategies for treating salt-sensitive hypertension and renal damage.

Keywords: epoxyeicosatrienoic acids, SHRSP, high-salt intake, renal damage, proteinuria, clofibrate, epoxygenase inhibition

\section{INTRODUCTION}

Salt-sensitivity is an important characteristic of a subgroup of humans with essential hypertension (Katori and Majima, 2008). In particular, high dietary salt increases the susceptibility of hypertensive patients to renal damage (Cowley and Roman, 1996). The stroke-prone substrain $\left(\mathrm{A}_{3} \mathrm{~N}\right)$ of the spontaneously hypertensive rat (SHR), or stroke-prone SHR (SHRSP), is a well-established model of genetic hypertension in which end-organ damage is highly salt-sensitive. Excess dietary salt dramatically increases the onset of stroke, myocardial infarction, and renal damage while only moderately elevating blood pressure (BP) further in SHRSP (Zuckerman et al., 1997). Previous studies in SHRSP using agents that interfere with the renin-angiotensin-aldosterone system have shown a dissociation between BP lowering and protection against

Abbreviations: AUDA, 2-(3-adamantan-1-yl-ureido)-dodecanoic acid; CYP, cytochrome P450; DHETs, dihydroxyeicosatrienoic acids; EETs, epoxyeicosatrienoic acids; ENaC, epithelial sodium channel; 20-HETE, 20hydroxyeicosatetraenoic acid; HS, high-salt; MS-PPOH, N-methylsulfonyl-6(2-propargyloxyphenyl)hexanamide; PAS, periodic acid-Schiff reagent; SBP, systolic blood pressure; sEH, soluble epoxide hydrolase; SHRSP, stroke-prone spontaneously hypertensive rat; SPRD, stroke-prone rodent diet; UPE, urinary protein excretion. salt-sensitive end-organ damage (Stier et al., 1991; Rocha et al., 1998) and have specifically implicated aldosterone as a major factor in the etiology of salt-sensitive kidney damage (Chander et al., 2003). Consistent with a pivotal pathophysiological role of aldosterone in the saline-drinking SHRSP and the ability of aldosterone to stimulate the epithelial sodium channel $(\mathrm{ENaC})$, Sepehrdad etal. $(2003,2004)$ found that amiloride and other agents that inhibit $\mathrm{ENaC}$ function offer protective effects against the development of proteinuria and renal microvascular damage. Interestingly, epoxyeicosatrienoic acids (EETs), cytochrome P450 (CYP) epoxygenase metabolites of arachidonic acid, have been shown to directly inhibit ENaC activity (Wei et al., 2004). EETs not only block the action of aldosterone at the level of the distal nephron, but they are also involved in the long-term regulation of $\mathrm{BP}$ and in the functional response of the kidney to high-salt (HS) diet (Liclican et al., 2008, 2009). Studies in experimental animal models also provide substantial evidence for EETs in the regulation in inflammation, fibrosis, and platelet aggregation (Node et al., 1999; Krotz et al., 2004). These properties of EETs enable them to serve in a variety of settings to protect and sustain both renal and systemic circulatory function. Therefore, 
we reasoned that interventions directed at induction or inhibition of epoxygenases responsible for the production of EETs might have a major impact on the pathologic renal changes that occur in saline-drinking SHRSP.

There are two pharmacological approaches that have been used to chronically elevate endogenous levels of EETs in order to evaluate their renal and vascular protective actions in vivo. One approach is to inhibit the conversion of EETs to their less active metabolites, dihydroxyeicosatrienoic acids (DHETs), by soluble epoxide hydrolase (sEH; Zeldin et al., 1995). We have shown that in vivo treatment with a sEH inhibitor, 2(3-adamantan-1-yl-ureido)-dodecanoic acid (AUDA), prevented the early salt-sensitive components of hypertension and kidney damage in saline-drinking SHRSP (Li et al., 2008). Another approach is to increase levels of EETs by inducing epoxygenases with fibric acid derivatives such as clofibrate, fenofibrate, and bezafibrate (Muller et al., 2004; Zhao et al., 2006). Fenofibrate has been shown to strongly induce renal protein expression of CYP2C23, a major CYP epoxygenase in the rat kidney, and increase renal epoxygenase activity (Muller et al., 2004). On the other hand, epoxygenases can be inhibited with $\mathrm{N}$-methylsulfonyl-6(2-propargyloxyphenyl)hexanamide (MS-PPOH), which has been identified as a potent and selective inhibitor of CYP-catalyzed arachidonate epoxidation both in vitro (Brand-Schieber et al., 2000) and in vivo (Liclican et al., 2009). We have previously reported that in vivo MS-PPOH treatment significantly reduced renal levels of EETs and rendered Dahl salt-resistant rats hypertensive (Liclican et al., 2009). In the present study, we hypothesized that epoxygenase stimulation would reduce $\mathrm{BP}$ and protect against renal damage in saline-drinking SHRSP, whereas inhibition of epoxygenase activity would increase $\mathrm{BP}$ and exacerbate renal damage in these animals.

\section{MATERIALS AND METHODS ANIMALS}

Six-week-old male SHRSP, bred from NIH stock derived originally from the SHRSP/A 3 N substrain, were obtained from Charles River Laboratories. Rats were given standard rodent diet (Purina Lab Chow \# 5001, 0.38\% $\mathrm{Na}^{+}$and 1.23\% K' $\mathrm{K}^{+}$Stier et al., 1989) and allowed tap water ad libitum. Animals were housed in a temperature-controlled room with a 12-h light/dark cycle and were used in accordance with NIH guidelines. The New York Medical College Institutional Animal Care and Use Committee approved all experimental protocols.

\section{CLOFIBRATE TREATMENT OF SHRSP}

SHRSP were maintained on stroke-prone rodent diet $(0.38 \% \mathrm{Na}$ and $0.71 \% \mathrm{~K}$, Zeigler Brothers, Gardners, PA, USA; Stier et al., 1989 ) and $1 \% \mathrm{NaCl}$ drinking solution starting at approximately 7 weeks of age. Clofibrate $(200 \mathrm{mg} / \mathrm{kg} /$ day, $n=7$; Sigma-Aldrich, St. Louis, MO, USA) or vehicle ( $0.5 \%$ methylcellulose, $n=5)$ administered once daily by gavage, was started 3 days prior to giving SHRSP 1\% NaCl drinking solution. Systolic BP (SBP) was measured weekly using tail-cuff plethysmography (CODA 2 noninvasive BP apparatus, Kent Scientific, Torrington, CT, USA). After 3 weeks of HS intake, animals were housed in metabolic cages and urine was collected for measurement of eicosanoids by
Liquid Chromatography/Mass Spectrometry/Mass Spectrometry (LC/MS/MS) analysis and urinary protein excretion (UPE) by the sulfosalicylic acid turbidity method (Stier et al., 1989). Rats were then anesthetized with sodium pentobarbital ( $65 \mathrm{mg} / \mathrm{kg}$, i.p.) and kidneys were excised and sections of cortex were snap frozen in liquid $\mathrm{N}_{2}$ for Western immunoblot analysis and the remaining kidney was placed in formalin for histological evaluation.

\section{MS-PPOH TREATMENT OF SHRSP}

The right jugular vein of 12 SHRSP was cannulated at 6 weeks of age as previously described (Liclican et al., 2009) and the animals were allowed 1 week for recovery. Animals were switched to strokeprone rodent diet and treated with MS-PPOH $(20 \mathrm{mg} / \mathrm{kg} / \mathrm{day}$, synthesized by Dr. John R. Falck, Texas Southwestern Medical Center, TX, USA; $n=7$ ) or vehicle ( $45 \%$ hydroxypropyl $\beta$ cyclodextrin, $1.5 \mathrm{ml} / \mathrm{kg} /$ day; $n=5$ ) as bolus injections into the jugular vein catheter twice per day, starting at 7 weeks of age. SHRSP were given $1 \% \mathrm{NaCl}$ to drink 3 days after the treatment with MS-PPOH was started. SBP was measured and urine was collected weekly after MS-PPOH treatment. UPE was measured weekly and the end point of the study was based on the occurrence of proteinuria, defined as a UPE of at least $20 \mathrm{mg} /$ day. At that time one animal from each group was anesthetized and tissue samples were obtained as described in the above section for clofibrate.

\section{ANALYSIS OF URINARY EICOSANOIDS}

An internal standard mixture containing 500 pg of $\mathrm{d}_{8}-11,12$-EET (Biomol, Plymouth Meeting, PA, USA), $\mathrm{d}_{11}-11,12$-DHET, and $\mathrm{d}_{6}$-20-hydroxyeicosatetraenoic acid (20-HETE; Cayman Chemical, Ann Arbor, MI, USA) was added to $2 \mathrm{ml}$ of urine and lipids were extracted with Bond Elut-Certify II columns (Varian, Lake Forest, CA, USA). Briefly, each sample was diluted with $2 \mathrm{ml}$ of $0.1 \mathrm{M}$ sodium acetate solution ( $\mathrm{pH} 7.0$ ) containing 5\% methanol, and the $\mathrm{pH}$ was adjusted to 6.0 with acetic acid. Columns were preconditioned with $2 \mathrm{ml}$ of methanol, followed by $2 \mathrm{ml}$ of $0.1 \mathrm{M}$ sodium acetate solution ( $\mathrm{pH} 7.0$ ) containing 5\% methanol before application of the urine samples. The columns were washed with $2 \mathrm{ml}$ of methanol-water ( $1: 1$ by volume), and urinary eicosanoids were eluted with $2 \mathrm{ml}$ of hexane: ethyl acetate (75:25 by volume) containing $1 \%$ acetic acid. The organic extracts were evaporated to dryness under $\mathrm{N}_{2}$ and reconstituted in $100 \mu \mathrm{L}$ of methanol (Rivera et al., 2004). Extracted samples were analyzed by a quadruple linear ion trap LC/MS/MS system (Q-Trap 3200) equipped with a Turbo $\mathrm{V}$ ion source operated in negative electrospray mode as previously described (Liclican et al., 2009). Data were analyzed with Analyst 4.02 software.

\section{WESTERN IMMUNOBLOT ANALYSIS}

Western immunoblotting was performed using an infrared fluorescence system (Odyssey; LI-COR Biosciences, Lincoln, NE, USA) as previously described (Liclican et al., 2008). Briefly, proteins were separated on a $10 \%$ SDS-PAGE gel and transferred to a polyvinylidene difluoride membrane. Membranes were blocked at room temperature for $1 \mathrm{~h}$ and incubated overnight at $4^{\circ} \mathrm{C}$ with the following primary antibodies: CYP2C23 (a generous gift from Dr. J. Capdevila, Vanderbilt University, TN, USA), CYP2C11 (Oxford 
Biomedical Research, Oxford, MI, USA), and CYP4A (Daiichi Chemical Co, Japan). The membranes were then incubated at room temperature for $1 \mathrm{~h}$ with corresponding IRDye secondary antibodies (LI-COR). Some membranes were stripped of bound antibodies and reprobed with a $\beta$-actin antibody. The intensity (densitometric units) ratio of target protein to $\beta$-actin on the same membrane was calculated and used for quantitative comparisons.

\section{HISTOLOGICAL EVALUATION}

Sections of kidney were fixed in 10\% neutral-buffered formalin and embedded in paraffin blocks. The sections were cut at a thickness of 2-4 $\mu \mathrm{m}$ and stained with hematoxylin and eosin or periodic acid-Schiff reagent (PAS) for examination by light microscopy as previously described (Zuckerman et al., 1997; Chander et al., 2003; Li et al., 2008). Histologic sections were examined by a renal pathologist (Praveen N. Chander) without the prior knowledge of the treatment. For renal vascular damage, the prevalence of pathologic lesions was quantified by counting the number of vascular profiles exhibiting fibrinoid degeneration/necrosis and proliferative vasculopathic lesions. Fibrinoid degeneration of vessels was defined as the absence of myocytic nuclei in conjunction with hypereosinophilia in an area of the vessel wall and/or accumulation of brightly, PAS positive globular material in the vessel wall. The data were expressed as the total number of vessels affected per field of 200 glomeruli (Li et al., 2008). For glomerular damage, glomerular necrotizing and proliferative lesions were counted and expressed also as per field of 200 glomeruli. Tubular protein casts were counted and expressed as number of tubules presenting casts per field of 200 glomeruli.

\section{STATISTICAL ANALYSES}

BP data were analyzed using a two-way ANOVA followed by Bonferroni post hoc test. Fisher's exact test was used to determine treatment effects on renal pathology. A Student's $t$-test was used to evaluate the protein expression. All other data were logtransformed to stabilize the variance before analyzing by Student's $t$-test. These data are displayed using the actual values. Differences were considered statistically significant at $P<0.05$. Data are expressed as means \pm SEM.

\section{RESULTS \\ EFFECT OF CLOFIBRATE TREATMENT ON SBP}

Figure 1 shows results for SBP. In vehicle-treated SHRSP, SBP progressively increased from $176 \pm 4 \mathrm{mmHg}$ at 1 week to $197 \pm 5 \mathrm{mmHg}$ at 3 weeks of the study $(P<0.01)$. SBP was significantly lower in clofibrate-treated SHRSP at each week of the study $(149 \pm 4 \mathrm{mmHg}$ at 1 week, $P<0.01$ vs. vehicle; $167 \pm 8 \mathrm{mmHg}$ at 3 weeks, $P<0.05$ vs. vehicle).

\section{EFFECT OF CLOFIBRATE TREATMENT ON UPE AND RENAL DAMAGE}

Pre-terminal UPE was significantly lower in SHRSP treated with clofibrate compared with vehicle-treated SHRSP $(P<0.001$, Figure 2H). Representative PAS-stained photomicrographs of renal cortical sections are provided in Figures 2A-F. In vehicletreated SHRSP, three weeks of HS intake produced moderate

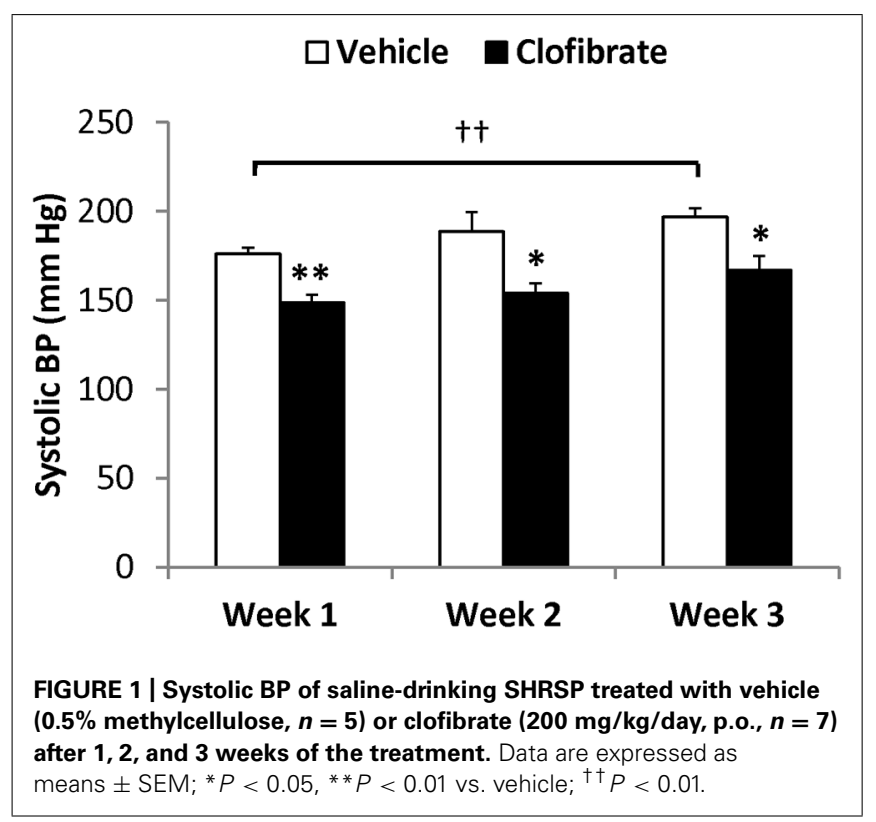

renal pathologic lesions, consisting primarily of vascular fibrinoid degeneration. The number of vessels exhibiting fibrinoid degeneration was significantly lower in clofibrate-treated SHRSP $(P<0.05$, Figure 2G). Leukocyte infiltration was present in the interstitium surrounding vessels that exhibited fibrinoid degeneration in vehicle-treated SHRSP and was abrogated in clofibratetreated SHRSP. Tubular protein casts were present in all (5/5) kidneys from vehicle-treated SHRSP whereas only one out of seven $(1 / 7)$ kidneys from clofibrate-treated SHRSP presented focal casts $(P<0.05)$. Consistent with reduced UPE and tubular protein casts, kidney sections from clofibrate-treated SHRSP showed less vascular hypertrophy and hyaline droplets in podocytes (arrowhead, Figure 2C) compared with vehicle-treated SHRSP.

\section{EFFECT OF CLOFIBRATE TREATMENT ON RENAL PROTEIN EXPRESSION OF EPOXYGENASE AND $\omega$-HYDROXYLASE}

Renal cortical protein expression of CYP2C23 was higher in clofibrate- compared with vehicle-treated SHRSP $(P<0.05$, Figure 3A), Renal cortical protein expression of CYP2C11, another epoxygenase expressed in rat kidney, did not differ between the groups, but tended to be higher in clofibrate- compared with vehicle-treated animals (Figure 3B). The renal cortical protein expression of CYP4A, the w-hydroxylase that oxidizes arachidonic acid to 20-HETE, was approximately twofold higher in clofibratethan vehicle-treated SHRSP $(P<0.01$, Figure $3 \mathrm{C})$.

\section{EFFECT OF CLOFIBRATE TREATMENT ON URINARY EXCRETION OF EETS AND DHETs}

Urinary excretion of EETs was approximately twofold higher in clofibrate- compared with vehicle-treated SHRSP $(P<0.005$, Figure 4), although the level of 14,15-EET was unaffected with clofibrate treatment (Table 1). Urinary excretion of DHETs was also significantly higher in clofibrate- compared with vehicletreated SHRSP $(P<0.01$, Figure 4$)$ as were the levels of 11, 12-DHET and 14, 15-DHET. Total urinary excretion of 

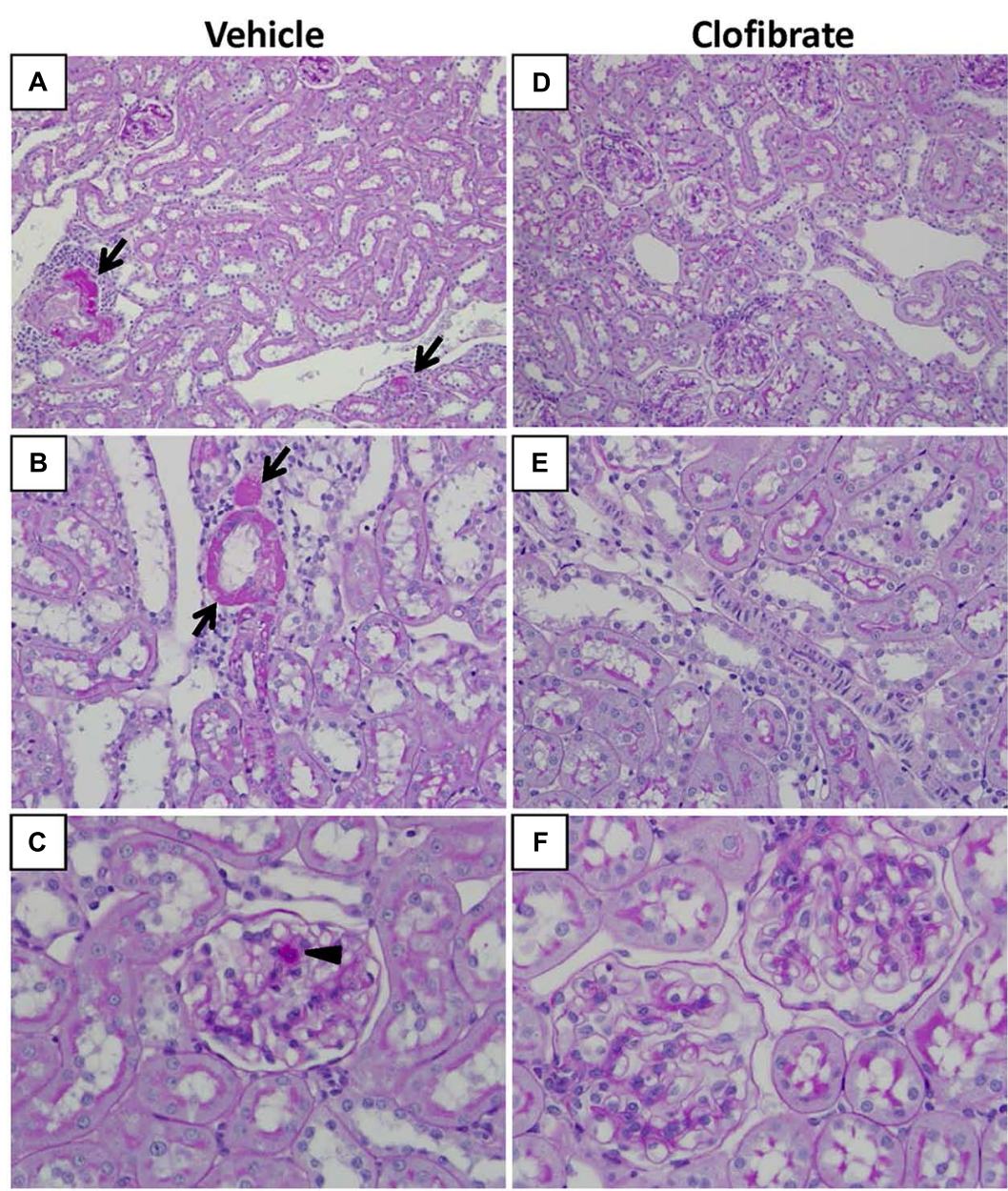

G

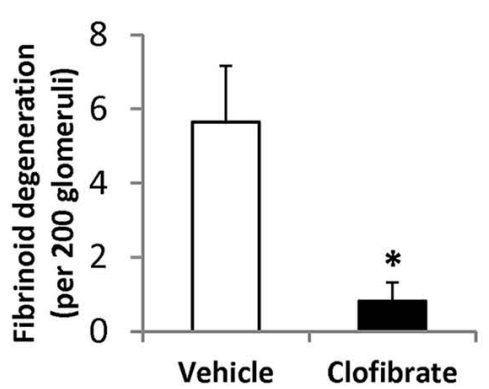

FIGURE 2 | (A-F) Representative PAS-stained photomicrographs of renal cortical sections from three of the vehicle-treated $(0.5 \%$ methylcellulose, $n=5, \mathbf{A}-\mathbf{C})$ and three of the clofibrate-treated ( $200 \mathrm{mg} / \mathrm{kg} /$ day, p.o., $n=7$, D-F) saline-drinking SHRSP at 3 weeks of the study. Renal sections from vehicle-treated SHRSP demonstrated focally prominent fibrinoid degeneration in vascular walls (arrows). The surrounding interstitium displays mononuclear leukocyte infiltration. Arrow head indicates prominent intracytoplasmic
H

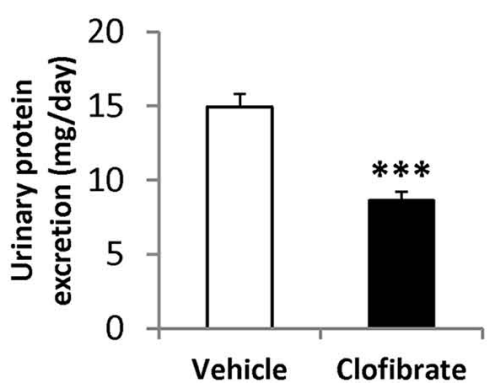

hyaline droplets in a glomerulus from a vehicle-treated SHRSP. Kidney sections from clofibrate-treated animals exhibited very scattered and mild vascular lesions. Magnification: $\times 20$ (A,D), $\times 40$ (B,E), or $\times 60(\mathbf{C}, \mathbf{F})$.

(G) Quantification of renal microvessels exhibiting fibrinoid degeneration per field of 200 glomeruli. (H) Urinary protein excretion of SHRSP treated with vehicle or clofibrate at 3 weeks of the study. Data are expressed as means \pm SEM; ${ }^{*} P<0.05,{ }^{* *} P<0.001$ vs. vehicle.
EETs + DHETs, an index of renal epoxygenase function, was higher in the clofibrate-treated group $(22.5 \pm 3.2 \mathrm{ng} /$ day $)$ than the vehicle-treated group $(11.8 \pm 0.3 \mathrm{ng} /$ day, $P<0.02)$. Urinary 20-HETE excretion was under the detection limit $(10 \mathrm{pg} / \mathrm{ml})$ of our LC/MS/MS.

\section{EFFECT OF MS-PPOH TREATMENT ON SBP}

The effects of in vivo MS-PPOH treatment on BP and kidney damage were examined in saline-drinking SHRSP. Animals were treated with MS-PPOH intravenously at a dosage of $20 \mathrm{mg} / \mathrm{kg} / \mathrm{day}$ (Huang et al., 2006; Liclican et al., 2009). MS-PPOH treatment 
A

\section{Cortex}
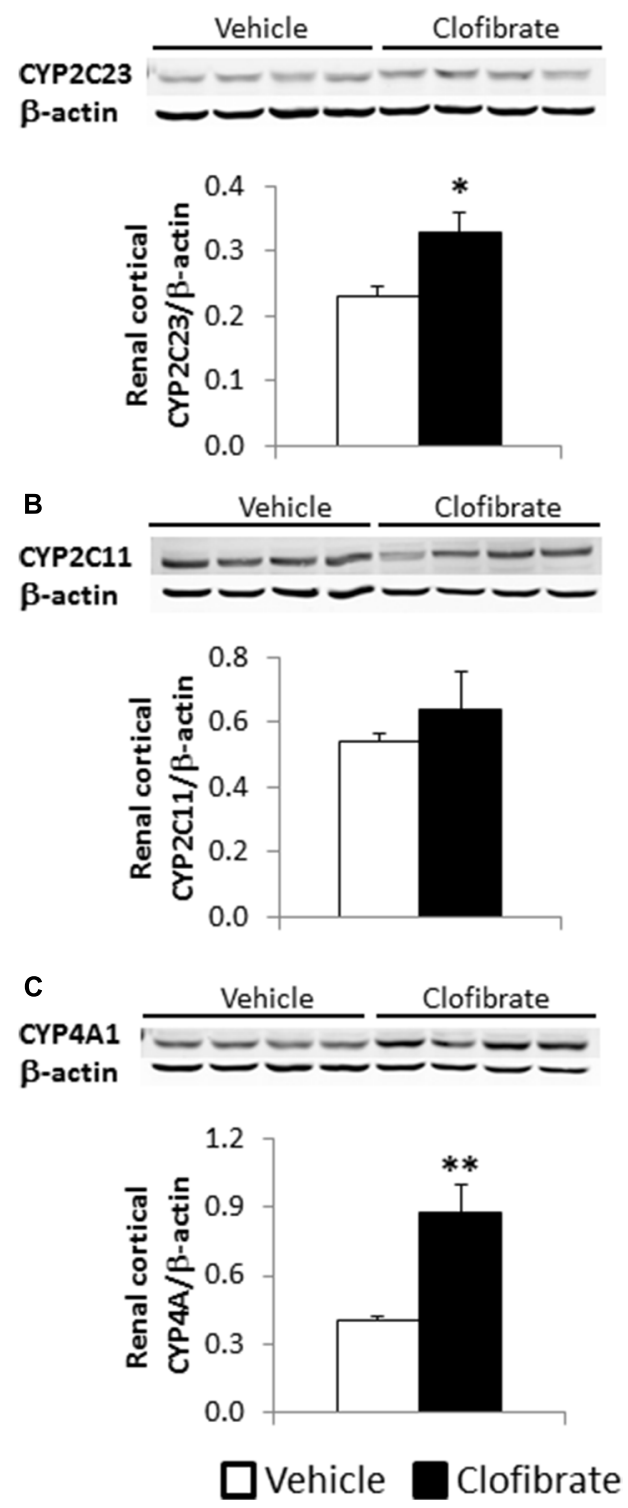

FIGURE 3 | Western blots and semi-quantitative summary of renal cortical protein expression of CYP2C23 (A, $48 \mathrm{kDa})$, CYP2C11 (B, $55 \mathrm{kDa}$ ) and CYP4A (C, $50 \mathrm{kDa})$ in saline-drinking SHRSP treated with vehicle $(0.05 \%$ methylcellulose $)$ or clofibrate $(200 \mathrm{mg} / \mathrm{kg} / \mathrm{day}$, p.o.) for 3 weeks. Renal cortical protein expression of CYP2C23 and CYP4A was significantly higher in clofibrate-treated SHRSP. Data are expressed as means \pm SEM; $n=4 .{ }^{*} P<0.05,{ }^{*} P<0.01$ vs. vehicle.

had negligible effects on SBP in saline-drinking SHRSP after 1 week, $160 \pm 7$ vs. $167 \pm 6 \mathrm{mmHg}$, or 2 weeks of treatment, $171 \pm 7$ vs. $175 \pm 8 \mathrm{mmHg}$, for vehicle vs. $\mathrm{MS}-\mathrm{PPOH}$, respectively.

\section{EFFECT OF MS-PPOH TREATMENT ON UPE AND RENAL HISTOLOGY}

SBP was not altered with MS-PPOH treatment; however, four out of seven (4/7) MS-PPOH-treated SHRSP developed proteinuria (defined as UPE $\geq 20 \mathrm{mg} /$ day). As expected, due to the young

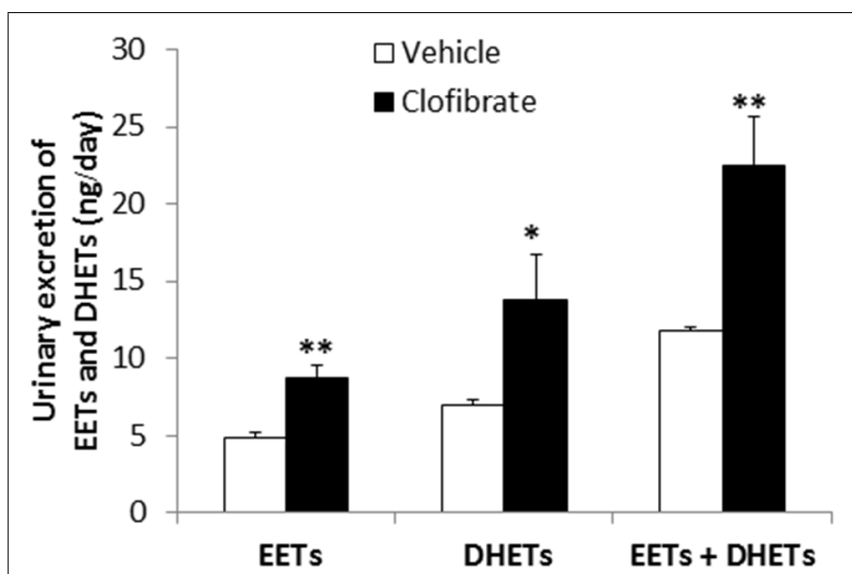

FIGURE 4 | Urinary excretion of EETs and DHETs in saline-drinking SHRSP treated with vehicle- $(0.5 \%$ methylcellulose, $n=5)$ or clofibrate (200 $\mathbf{~ m g / k g / d a y , ~ p . o . , ~} \boldsymbol{n}=\mathbf{7}$ ) for $\mathbf{3}$ weeks. Urinary excretion of EETs and/or DHETs was significantly higher in clofibrate-treated SHRSP. The sum of EETs and DHETs, an index of epoxygenase function, was also significantly increased with clofibrate treatment. Data were

log-transformed to stabilize the variance before analyzing by Student's

$t$-test. The actual values are displayed and data are expressed as means $\pm \mathrm{SEM}$; ${ }^{*} P<0.01,{ }^{*} P<0.005$ vs. vehicle.

age and short period of HS intake, none (0/5) of the vehicletreated SHRSP showed proteinuria. Overall UPE was higher in MS-PPOH-treated SHRSP compared with vehicle-treated animals $(P<0.01$, Figure $\mathbf{5 F})$. Figures $\mathbf{5 A} \mathbf{\mathbf { B }} \mathbf{B}$ shows representative photomicrographs of PAS-stained kidney sections from vehicletreated SHRSP, which demonstrated only scattered and mild renal vascular changes. In contrast, kidneys from $\mathrm{MS}-\mathrm{PPOH}$-treated SHRSP exhibited widespread lesions of malignant nephrosclerosis characterized by segmental to circumferential mural fibrinoid necrosis of microvessels with proliferative arteriopathy, which typically is seen in much older SHRSP with prolonged HS intake (Figures 5C,D). Proliferative lesions, which signify more severe microvascular damage, were present in five out of seven (5/7) kidneys from MS-PPOH-treated SHRSP, whereas none $(0 / 5)$ of the vehicle-treated SHRSP developed such lesions $(P<0.05)$. The number of vessels exhibiting fibrinoid necrosis and/or proliferative

Table 1 | Effect of clofibrate $(200 \mathrm{mg} / \mathrm{kg} / \mathrm{day}, \mathrm{p.o}$.) on urinary excretion of EETs and DHETs (ng/day) in saline-drinking SHRSP.

\begin{tabular}{lllll}
\hline & $\mathbf{1 4 , 1 5}-\mathrm{EET}$ & $\mathbf{1 1 , 1 2 - E E T}$ & $\mathbf{8 , 9}-\mathrm{EET}$ & $\mathbf{5 , 6 - E E T}$ \\
\hline Vehicle & $1.64 \pm 0.15$ & $1.26 \pm 0.05$ & $0.90 \pm 0.13$ & $1.03 \pm 0.20$ \\
Clofibrate & $2.64 \pm 0.46$ & $2.31 \pm 0.26^{* *}$ & $1.47 \pm 0.16^{*}$ & $2.26 \pm 0.33^{*}$ \\
\hline & $\mathbf{1 4 , 1 5 - D H E T}$ & $\mathbf{1 1 , 1 2 - D H E T}$ & $\mathbf{8 , 9}-\mathrm{DHET}$ & $\mathbf{5 , 6 - D H E T}$ \\
\hline Vehicle & $3.34 \pm 0.27$ & $0.40 \pm 0.04$ & $0.62 \pm 0.09$ & $2.60 \pm 0.41$ \\
Clofibrate & $7.37 \pm 1.52^{*}$ & $0.76 \pm 0.12^{*}$ & $0.98 \pm 0.48$ & $4.68 \pm 1.03$
\end{tabular}

Data were log-transformed to stabilize the variance before analyzing by Student's $t$-test. The actual values are displayed and data are expressed as means \pm SEM; $n=5$ in vehicle group and $n=7$ in clofibrate group; ${ }^{*} P<0.05,{ }^{*} P<0.002$ vs. vehicle. 

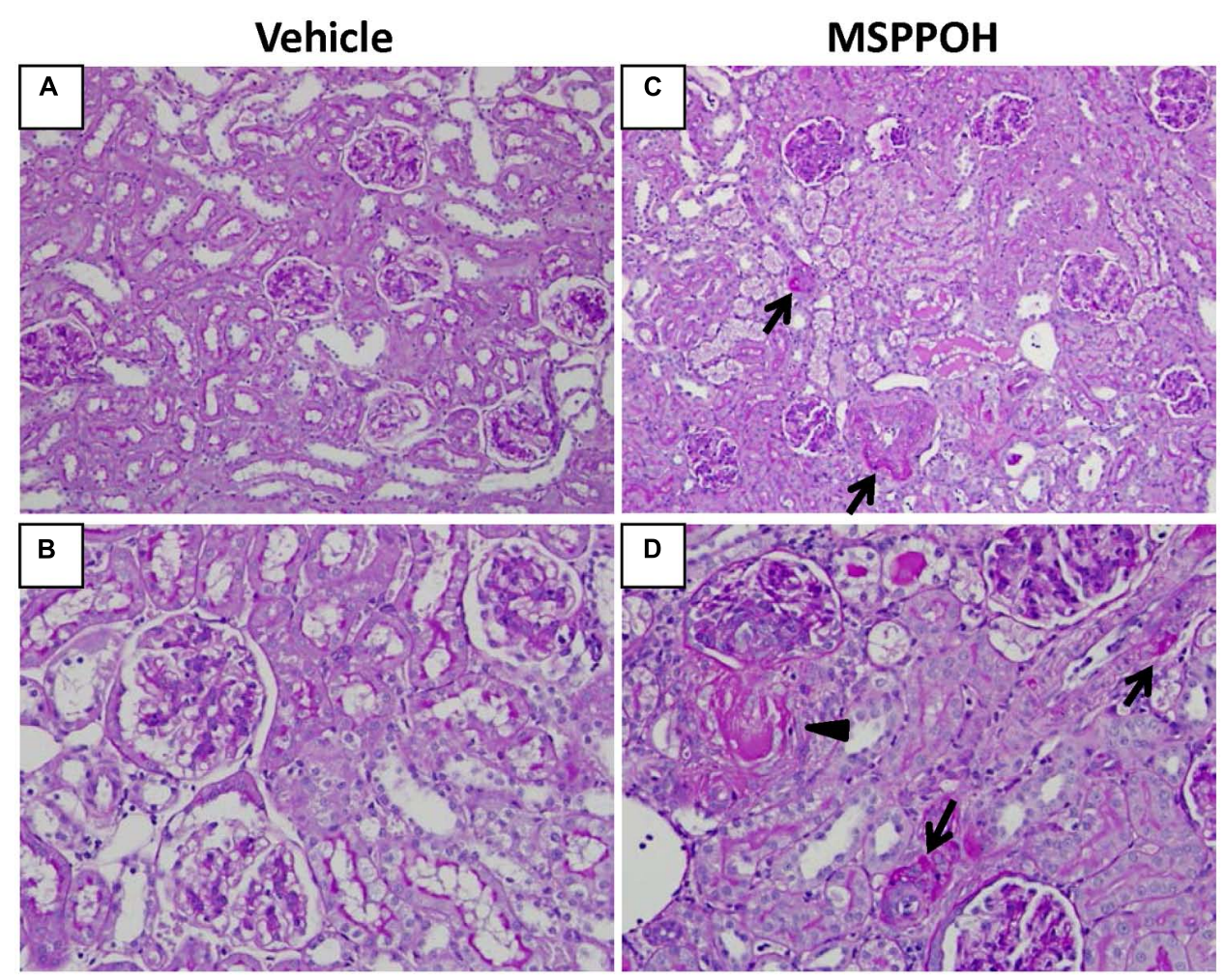

E

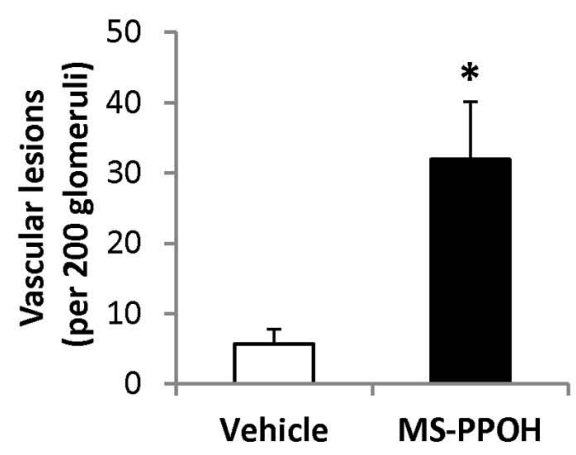

FIGURE $\mathbf{5}$ | (A-D) Representative PAS-stained photomicrographs of renal cortex from two saline-drinking SHRSP treated with vehicle $(45 \%$ hydroxypropyl $\beta$-cyclodextrin, $n=5 ; \mathbf{A}, \mathbf{B})$ and two saline-drinking SHRSP treated with MS-PPOH $(20 \mathrm{mg} / \mathrm{kg} /$ day, i.v., $n=7 ; \mathbf{C , D})$ for 2 weeks. Kidney sections from vehicle-treated SHRSP demonstrated very scattered and mild early microvascular lesions whereas kidney sections from MS-PPOH-treated SHRSP exhibited widespread and well-established lesions of malignant nephrosclerosis characterized by segmental to circumferential fibrinoid necrosis of microvessels (arrows). Arrow head points to a proliferative
$\mathbf{F}$

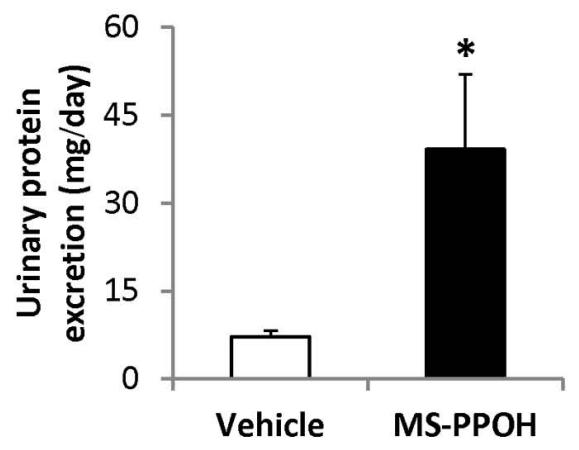

microvascular lesion; the adjacent glomerulus also shows segmental necrotizing and proliferative lesions from a MS-PPOH-treated SHRSP. These animals also exhibited focal but significant protein casts which were commensurate with proteinuria. Protein casts were generally absent in vehicle-treated animals. Magnification: $\times 20(\mathbf{A}, \mathbf{C})$ or $\times 40$ (B,D).

(E) Quantification of renal microvessels exhibiting fibrinoid degeneration and/or proliferative lesions per field of 200 glomeruli. (F) Urinary protein excretion of SHRSP treated with vehicle or MS-PPOH for 2 weeks. Data are expressed as means \pm SEM; ${ }^{*} P<0.05$ vs. vehicle. lesions (Figure 5E) was markedly increased in kidneys from MS-PPOH-treated SHRSP compared with vehicle-treated SHRSP $(P<0.05)$. In addition to vascular damage, four out of seven (4/7) kidneys from MS-PPOH-treated SHRSP exhibited glomerular necrotizing and proliferative lesions, whereas none $(0 / 5)$ of the vehicle-treated SHRSP developed glomerular lesions. Commensurate with UPE, the number of tubules presenting protein casts was markedly increased in kidneys from MS-PPOH-treated
SHRSP ( $11 \pm 3$ per field of 200 glomeruli) compared with vehicle $(2 \pm 1$ per field of 200 glomeruli, $P<0.05)$.

\section{EFFECT OF MS-PPOH TREATMENT ON URINARY EXCRETION OF EETS AND DHETs}

Compared with vehicle-treated SHRSP, urinary excretion of EETs and DHETS was not different in MS-PPOH-treated SHRSP at 2 weeks of treatment (Figure 6). Total urinary excretion 


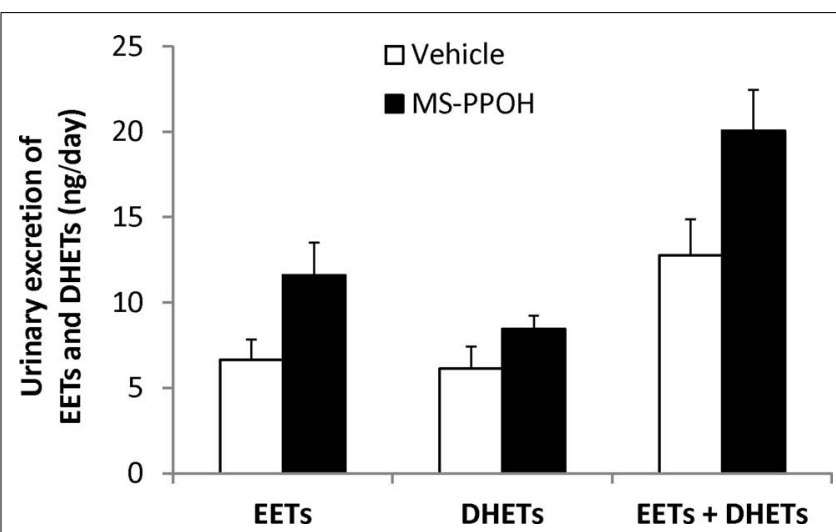

FIGURE 6 | Urinary excretion of EETs and DHETs in vehicle- $145 \%$ hydroxypropyl $\beta$-cyclodextrin, $n=5)$ and MS-PPOH- $(20 \mathrm{mg} / \mathrm{kg} / \mathrm{day}$, i.v., $n=7$ ) treated SHRSP at 2 weeks of treatment. Urinary EET excretion and the sum of EETs + DHETs tended to be higher in MS-PPOH treated SHRSP, but these did not reach statistical significance. Data were log-transformed to stabilize the variance before analyzing by Student's $t$-test. The actual values are displayed and data are expressed as means \pm SEM.

of EETs + DHETs tended to be higher in the MS-PPOH(12.8 $\pm 2.1 \mathrm{ng} /$ day) compared with vehicle-treated group $(20.1 \pm 2.4 \mathrm{ng} /$ day $)$. However, this also did not reach statistical significance $(P=0.052)$. None of the urinary levels of individual EET and DHET regioisomers were significantly altered with MS-PPOH treatment (Table 2).

\section{DISCUSSION}

In the present study, we used clofibrate as a pharmacological approach to elevate EET levels in SHRSP. In agreement with our previous study using a $s E H$ inhibitor in young saline-drinking SHRSP (Li et al., 2008), treatment of clofibrate significantly reduced SBP and produced a renal protective effect. Fibrates have been reported to lower BP in several salt-loaded genetic models of hypertension (Roman et al., 1993; Shatara et al., 2000; Zhou et al., 2008), but have no effect on BP in normotensive SpragueDawley rats (Shatara et al., 2000). More importantly, clofibrate prevented DOCA-salt induced increases in BP in mice, indicating that the $\mathrm{BP}$ lowering effect of clofibrate is not necessarily

Table 2 | Effect of MS-PPOH ( $20 \mathrm{mg} / \mathrm{kg} /$ day, i.v.) on urinary excretion of EETs and DHETs (ng/day) in saline-drinking SHRSP.

\begin{tabular}{lllll}
\hline & $\mathbf{1 4 , 1 5}-$ EET & $\mathbf{1 1 , 1 2 - E E T}$ & $\mathbf{8 , 9 - E E T}$ & $\mathbf{5 , 6 - E E T}$ \\
\hline Control & $2.50 \pm 0.52$ & $1.79 \pm 0.26$ & $1.42 \pm 0.19$ & $0.93 \pm 0.25$ \\
MS-PPOH & $4.71 \pm 0.76$ & $3.05 \pm 0.51$ & $2.78 \pm 0.54$ & $1.07 \pm 0.24$ \\
\hline & $\mathbf{1 4 , 1 5}$-DHET & $\mathbf{1 1 , 1 2 - D H E T}$ & $\mathbf{8 , 9}$-DHET & $\mathbf{5 , 6 - D H E T}$ \\
\hline Control & $2.62 \pm 0.66$ & $0.55 \pm 0.10$ & $0.48 \pm 0.07$ & $2.47 \pm 0.51$ \\
MS-PPOH & $3.34 \pm 0.51$ & $1.10 \pm 0.22$ & $0.69 \pm 0.11$ & $3.34 \pm 0.34$
\end{tabular}

Data were log-transformed to stabilize the variance before analyzing by Student's t-test. The actual values are displayed and data are expressed as means $\pm S E M$; $n=5$ in control group and $n=7$ in MS-PPOH group. secondary to a suppression of endogenous mineralocorticoid levels (Zhou etal., 2008). One of the underling mechanisms for the antihypertensive actions of fibrates has been suggested to be due to increased synthesis of 20-HETE (Alonso-Galicia et al., 1998; Zhou etal., 2008). However, fibric acid derivatives have also been reported to increase epoxygenase activity and EET production. Fenofibrate reduced BP and increased epoxygenase expression in double transgenic rats overexpressing both the human renin and angiotensinogen genes (Muller et al., 2004), as well as in Sprague-Dawley rats fed a high-fat diet (Huang et al., 2007) and Zucker diabetic fatty rats (Zhao etal., 2006; Zhao and Li, 2008). Fenofibrate treatment prevented brain and renal damage and reduced inflammation and oxidative stress of SHRSP (Gelosa et al., 2010). In the present study, renal cortical CYP2C23 protein expression and urinary excretion of EETs and DHETs were significantly increased by treatment with clofibrate. Clofibrate has also been reported to upregulate expression of cerebral CYP2C11 of SHRSP; however, CYP2C23 protein was not detected (Ying et al., 2008). These observations, taken together with our previous finding that $\mathrm{sEH}$ inhibition for two weeks prevented HS-induced increases in SBP in young SHRSP, suggest that EETs may contribute importantly to the BP lowering effect of clofibrate in saline-drinking SHRSP.

SHRSP develop proteinuria, glomerular damage, and renal microvascular lesions characteristic of thrombotic microangiopathy in malignant nephrosclerosis with age. These renal pathologic changes are markedly accelerated by salt-loading and standard rodent diet when started at approximately 7 weeks of age. SHRSP manifest severe renal damage after 4 weeks on HS intake and die of strokes, primarily hemorrhagic infarcts, by 13-15 weeks of age (6-8 weeks of HS intake; Stier et al., 1991, 1993). In the present study, 3 weeks of HS intake produced mild to moderated vascular fibrinoid degeneration in vehicletreated SHRSP which was consistent with the progression to severe renal damage (almost 15\% of glomeruli and 20 vascular lesions per field of 100 glomeruli) after 4 weeks of HS intake in SHRSP (Zuckerman et al., 1997). Clofibrate treatment reduced UPE and markedly diminished renal glomerular and vascular damage. These results are consistent with our finding that sEH inhibition ameliorated early salt-sensitive renal damage in saline-drinking SHRSP, which supports a protective role for EETs against salt-sensitive renal damage ( $\mathrm{Li}$ et al., 2008). Fibrates have been reported to ameliorate renal damage in other animal models. In double transgenic rats overexpressing both human renin and angiotensinogen genes, fenofibrate reduced renal collagen IV expression and leukocyte infiltration (Muller et al., 2004). Fenofibrate also attenuated glomerular hypertrophy and collagen accumulation in Zucker diabetic fatty rats (Zhao and Li, 2008). Treatment with fenofibrate has been reported to reduce the number of abnormal glomeruli and to diminish the degree of mesangial expansion and glomerulosclerosis in Dahl salt-sensitive rats (Wilson et al., 1998). It is well known that EETs possess anti-inflammatory and antiproliferative activity in vascular smooth muscle (McGiff and Ferreri, 2007). Recently, 8,9-EET has been shown to prevent the focal segmental glomerulosclerosis-induced increase in 
glomerular albumin permeability in vitro (Sharma et al., 2009). Thus, we reasoned that the reduction of UPE and renal damage by clofibrate may be related to the increase in levels of EETs. To be noted, in the present study, since BP was appreciably lowered by clofibrate treatment, we cannot separate direct vasculoprotective effects of increasing EET levels from BP lowering effects on the development of renal damage in these rats. Clofibrate treatment was, however, reported to increase cerebral blood flow, prevent stroke and prolong survival of SHRSP (Ying et al., 2008).

Although we found an increase in CYP4A protein expression, unlike urinary EETs, 20-HETE was undetectable in the urine in either vehicle- or clofibrate-treated SHRSP as measured by LC/MS/MS. Like 20-HETE, EETs possess diuretic and natriuretic properties (Houillier et al., 1996; Wei et al., 2004). However, unlike 20-HETE which can cause vasoconstriction (McGiff and Ferreri, 2007), EETs produce vasodilatation of renal arterioles and may serve as the endothelium-derived hyperpolarizing factor in the vasculature (Eckman etal., 1998; Huang et al., 2005). 20-HETE has also been reported to play a role in controlling the glomerular permeability barrier to albumin, which mimics the effect of 8,9-EET at the glomerular permeability barrier (McCarthy et al., 2005). A recent study demonstrated that introgression of the CYP4A genes from Lewis rats into the Dahl salt-sensitive rats increased renal formation of 20-HETE and attenuated the development of hypertension and renal disease (Williams et al., 2008). In the present study, although urinary 20-HETE was undetectable, renal cortical CYP4A protein expression was induced by clofibrate treatment in saline-drinking SHRSP. Thus, we cannot rule out a possible contribution of CYP4A/20-HETE to the renal protective effects of clofibrate. Indeed, it may be the combined effects of EETs and 20-HETE which is responsible for the marked renal protection in saline-drinking SHRSP as both EETs and 20HETE promote sodium excretion and act to limit glomerular proteinuria.

We also examined inhibition of epoxygenase to determine if loss of EETs would promote end-organ damage in salinedrinking SHRSP. MS-PPOH has been identified as a potent and selective inhibitor of epoxygenases in vitro and in vivo (BrandSchieber et al., 2000). Rat renal microsomal epoxygenase activity was inhibited for up to $6 \mathrm{~h}$ after a single i.v. bolus injection of MS-PPOH (5 mg; Brand-Schieber et al., 2000) and we have previously shown that treatment with MS-PPOH $(20 \mathrm{mg} / \mathrm{kg} / \mathrm{day}$, i.v.) for 6 days significantly reduced renal levels of EETs in Dahl salt-resistant rats on $2 \% \mathrm{NaCl}$ drinking solution (Liclican et al., 2009). Consistent with this hypothesis, we found that in vivo epoxygenase inhibition with MS-PPOH in young SHRSP increased UPE and accelerated the development of renal damage consistent with malignant nephrosclerosis, as typically seen in older SHRSP after at least 4 weeks of salt-loading (Rocha et al., 1998). Vehicle treatment was associated with only mild renal damage as we have seen previously with 2 weeks of HS intake (Zuckerman et al., 1997; Li et al., 2008). Moreover, the microvascular damage with MS-PPOH treatment was significantly greater than that present in the vehicle-treated SHRSP in the clofibrate study, despite the fact that these animals were younger ( 9 vs. 10 weeks of age) and received HS intake for a shorter period of time than in the clofibrate study (11 days vs. 3 weeks). The data therefore suggest that EETs play a protective role against the development of salt-sensitive renal damage in saline-drinking SHRSP.

It has been previously reported that in vivo MS-PPOH treatment significantly increased BP, renal vascular resistance and sodium balance in pregnant rats (Huang et al., 2006) and increased BP in Dahl salt-resistant rats on HS intake (Liclican et al., 2009). In the present study, however, SBP was not further elevated with MS-PPOH treatment. This discrepancy may reflect a strain difference or a difference in pre-treatment BP, since the earlier two animal models were normotensive whereas SHRSP were hypertensive before the MS-PPOH treatment. Although SBP was not increased with MS-PPOH treatment in the present study, our findings are consistent with the notion that endogenous EETs may serve a renal vascular protective role independent of an effect on BP (Olearczyk et al., 2009).

Urinary excretion of EETs and DHETs was not reduced with MS-PPOH treatment in the present study. However, the appearance of heavy proteinuria in MS-PPOH-treated SHRSP may have masked this affect. The majority of plasma EETs bind to plasma albumin non-covalently in the form of fatty acid-albumin complex (Saifer and Goldman, 1961). Therefore, the EETs that bind to urinary albumin can be extracted by our method without the requirement for alkaline hydrolysis. Since some MS-PPOHtreated animals developed moderate to heavy proteinuria, EETs that bind with urinary albumin may have masked the reduction of EETs by epoxygenase inhibition with MS-PPOH. In addition to inhibition of epoxygenases, MS-PPOH has been reported to compete for the binding of a radiolabeled EET antagonist to cell membranes and inhibit 14,15-EET-induced relaxation of bovine coronary artery, suggesting a secondary mechanism by which MS$\mathrm{PPOH}$ has an inhibitory action on the epoxygenase-EET pathway (Chen et al., 2009).

In conclusion, clofibrate treatment reduced SBP, UPE, and renal damage in saline-drinking SHRSP, effects that may be related to increased levels of EETs as reflected by up-regulation of renal cortical epoxygenase expression and increased urinary excretion of EETs. In contrast, the CYP450 epoxygenase inhibitor, MS-PPOH, increased UPE and accelerated the development of renal damage in saline-drinking SHRSP without altering urinary EET levels. Together with our previous finding that sEH inhibition prevented early microvascular damage in saline-drinking SHRSP (Li et al., 2008), these results suggest that pharmacological manipulation of epoxygenase results in divergent effects on renal damage such that inhibition promotes injury and increasing EET synthesis reduces injury. Interventions to increase renal EET levels may provide therapeutic strategies for treating salt-sensitive hypertension and renal damage.

\section{ACKNOWLEDGMENTS}

Financial support provided by grants from the National Institutes of Health DK69687, DK38226, HL-25394, and GM31278 and the Robert A. Welch Foundation (GL625910). We thank Anabel B. Doumad and Elizabeth C. Villegas for their excellent technical assistance and Katherine Gotlinger for performing the LC/MS/MS study. 


\section{REFERENCES}

Alonso-Galicia, M., Frohlich, B., and Roman, R. J. (1998). Induction of P4504A activity improves pressure-natriuresis in Dahl S rats. Hypertension 31, 232-236. doi: 10.1161/01.HYP.31.1.232

Brand-Schieber, E., Falck, J. F., and Schwartzman, M. (2000). Selective inhibition of arachidonic acid epoxidation in vivo. J. Physiol. Pharmacol. 51, 655-672.

Chander, P. N., Rocha, R., Ranaudo, J., Singh, G., Zuckerman, A., and Stier, C. T. Jr. (2003). Aldosterone plays a pivotal role in the pathogenesis of thrombotic microangiopathy in SHRSP. J. Am. Soc. Nephrol. 14, 1990-1997. doi: 10.1097/01.ASN.0000078960.15147.05

Chen, Y., Falck, J. R., Tuniki, V. R., and Campbell, W. B. (2009). 20-125Iodo-14, 15-epoxyeicosa-5(Z)-enoic acid: a high-affinity radioligand used to characterize the epoxyeicosatrienoic acid antagonist binding site. J. Pharmacol. Exp. Ther. 331, 1137-1145. doi: 10.1124/jpet.109.157818

Cowley, A. W. Jr., and Roman, R. J. (1996). The role of the kidney in hypertension. J. Am. Med. Assoc. 275, 1581-1589. doi: 10.1001/jama.1996.03530440 061038

Eckman, D. M., Hopkins, N., McBride, C., and Keef, K. D. (1998). Endotheliumdependent relaxation and hyperpolarization in guinea-pig coronary artery: role of epoxyeicosatrienoic acid. Br. J. Pharmacol. 124, 181-189. doi: 10.1038/sj.bjp.0701778

Gelosa, P., Banfi, C., Gianella, A., Brioschi, M., Pignieri, A., Nobili, E., et al. (2010). Peroxisome proliferator-activated receptor $\alpha$ agonism prevents renal damage and the oxidative stress and inflammatory processes affecting the brains of stroke-prone rats. J. Pharmacol. Exp. Ther. 335, 324-331. doi: 10.1124/jpet.110. 171090

Houillier, P., Chambrey, R., Achard, J. M., Froissart, M., Poggioli, J., and Paillard, M. (1996). Signaling pathways in the biphasic effect of angiotensin II on apical $\mathrm{Na} / \mathrm{H}$ antiport activity in proximal tubule. Kidney Int. 50, 1496-1505. doi: 10.1038/ki.1996.464

Huang, A., Sun, D., Jacobson, A., Carroll, M. A., Falck, J. R., and Kaley, G. (2005). Epoxyeicosatrienoic acids are released to mediate shear stress-dependent hyperpolarization of arteriolar smooth muscle. Circ. Res. 96, 376-383. doi: 10.1161/01.RES.0000155332.17783.26

Huang, H., Chang, H. H., Xu, Y., Reddy, D. S., Du, J., Zhou, Y., et al. (2006). Epoxyeicosatrienoic acid inhibition alters renal hemodynamics during pregnancy. Exp. Biol. Med. (Maywood) 231, 1744-1752.

Huang, H., Morisseau, C., Wang, J., Yang, T., Falck, J. R., Hammock, B. D., et al. (2007). Increasing or stabilizing renal epoxyeicosatrienoic acid production attenuates abnormal renal function and hypertension in obese rats. Am. J. Physiol. Renal Physiol. 293, F342-F349. doi: 10.1152/ajprenal.00004. 2007

Katori, M., and Majima, M. (2008). Are all individuals equally sensitive in the blood pressure to high salt intake? (Review article). Acta Physiol. Hung. 95, 247-265. doi: 10.1556/APhysiol.95.2008.3.2

Krotz, F., Riexinger, T., Buerkle, M. A., Nithipatikom, K., Gloe, T., Sohn, H. Y., et al. (2004). Membrane-potential-dependent inhibition of platelet adhesion to endothelial cells by epoxyeicosatrienoic acids. Arterioscler. Thromb. Vasc. Biol. 24, 595-600. doi: 10.1161/01.ATV.0000116219. $09040.8 \mathrm{c}$

Li, J., Carroll, M. A., Chander, P. N., Falck, J. R., Sangras, B., and Stier, C. T. (2008). Soluble epoxide hydrolase inhibitor, AUDA, prevents early salt-sensitive hypertension. Front. Biosci. 13:3480-3487. doi: 10.2741/ 2942

Liclican, E. L., Doumad, A. B., Wang, J., Li, J., Falck, J. R., Stier, C. T. Jr., et al. (2009). Inhibition of the adenosine2A receptor-epoxyeicosatrienoic acid pathway renders Dahl salt-resistant rats hypertensive. Hypertension 54, 1284-1290. doi: 10.1161/HYPERTENSIONAHA.108.123570

Liclican, E. L., McGiff, J. C., Falck, J. R., and Carroll, M. A. (2008). Failure to upregulate the adenosine $2 \mathrm{~A}$ receptor-epoxyeicosatrienoic acid pathway contributes to the development of hypertension in Dahl salt-sensitive rats. Am. J. Physiol. Renal Physiol. 295, F1696-F1704. doi: 10.1152/ajprenal.90502. 2008

McCarthy, E. T., Sharma, R., and Sharma, M. (2005). Protective effect of 20-hydroxyeicosatetraenoic acid (20-HETE) on glomerular protein permeability barrier. Kidney Int. 67, 152-156. doi: 10.1111/j.1523-1755.2005. 00065.x
McGiff, J. C., and Ferreri, N. R. (2007). "Eicosanoids and the kidney," in Seldin and Giebisch's the Kidney, Chap. 14, eds R. J. Alpern and S. C. Hebert (Burlington, MA: Elsevier), 359-384.

Muller, D. N., Theuer, J., Shagdarsuren, E., Kaergel, E., Honeck, H., Park, J. K., etal. (2004). A peroxisome proliferator-activated receptor- $\alpha$ activator induces renal CYP2C23 activity and protects from angiotensin II-induced renal injury. Am. J. Pathol. 164, 521-532. doi: 10.1016/S0002-9440(10) 63142-2

Node, K., Huo, Y., Ruan, X., Yang, B., Spiecker, M., Ley, K., et al. (1999). Antiinflammatory properties of cytochrome $\mathrm{P} 450$ epoxygenase-derived eicosanoids. Science 285, 1276-1279. doi: 10.1126/science.285.5431.1276

Olearczyk, J. J., Quigley, J. E., Mitchell, B. C., Yamamoto, T., Kim, I. H., Newman, J. W., et al. (2009). Administration of a substituted adamantyl urea inhibitor of soluble epoxide hydrolase protects the kidney from damage in hypertensive Goto-Kakizaki rats. Clin. Sci. (Lond.) 116, 61-70. doi: 10.1042/CS20080039

Rivera, J., Ward, N., Hodgson, J., Puddey, I. B., Falck, J. R., and Croft, K. D. (2004). Measurement of 20-hydroxyeicosatetraenoic acid in human urine by gas chromatography-mass spectrometry. Clin. Chem. 50, 224-226. doi: 10.1373/clinchem.2003.025775

Rocha, R., Chander, P. N., Khanna, K., Zuckerman, A., and Stier, C. T. Jr. (1998). Mineralocorticoid blockade reduces vascular injury in stroke-prone hypertensive rats. Hypertension 31, 451-458. doi: 10.1161/01.HYP.31.1.451

Roman, R. J., Ma, Y. H., Frohlich, B., and Markham, B. (1993). Clofibrate prevents the development of hypertension in Dahl salt-sensitive rats. Hypertension 21, 985-988. doi: 10.1161/01.HYP.21.6.985

Saifer, A., and Goldman, L. (1961). The free fatty acids bound to human serum albumin. J. Lipid Res. 2, 268-270.

Sepehrdad, R., Chander, P. N., Oruene, A., Rosenfeld, L., Levine, S., and Stier, C. T. Jr. (2003). Amiloride reduces stroke and renal injury in stroke-prone hypertensive rats. Am. J. Hypertens. 16, 312-318. doi: 10.1016/S0895-7061(03) 00006-2

Sepehrdad, R., Chander, P. N., Singh, G., and Stier, C. T. Jr. (2004). Sodium transport antagonism reduces thrombotic microangiopathy in stroke-prone spontaneously hypertensive rats. Am. J. Physiol. Renal Physiol. 286, F1185-F1192. doi: 10.1152/ajprenal.00355.2003

Sharma, M., McCarthy, E. T., Reddy, D. S., Patel, P. K., Savin, V. J., Medhora, M., et al. (2009). 8,9-Epoxyeicosatrienoic acid protects the glomerular filtration barrier. Prostaglandins Other Lipid Mediat. 89, 43-51. doi: 10.1016/j.prostaglandins.2009.04.004

Shatara, R. K., Quest, D. W., and Wilson, T. W. (2000). Fenofibrate lowers blood pressure in two genetic models of hypertension. Can. J. Physiol. Pharmacol. 78, 367-371. doi: 10.1139/y99-149

Stier, C. T. Jr., Adler, L. A., Levine, S., and Chander, P. N. (1993). Stroke prevention by losartan in stroke-prone spontaneously hypertensive rats. J. Hypertens. Suppl. 11, S37-S42.

Stier, C. T. Jr., Benter, I. F., Ahmad, S., Zuo, H. L., Selig, N., Roethel, S., et al. (1989). Enalapril prevents stroke and kidney dysfunction in salt-loaded stroke-prone spontaneously hypertensive rats. Hypertension 13, 115-121. doi: 10.1161/01.HYP.13.2.115

Stier, C. T. Jr., Chander, P., Gutstein, W. H., Levine, S., and Itskovitz, H. D. (1991). Therapeutic benefit of captopril in salt-loaded stroke-prone spontaneously hypertensive rats is independent of hypotensive effect. Am. J. Hypertens. 4, 680-687. doi: 10.1093/ajh/4.8.680

Wei, Y., Lin, D. H., Kemp, R., Yaddanapudi, G. S., Nasjletti, A., Falck, J. R., et al. (2004). Arachidonic acid inhibits epithelial Na channel via cytochrome P450 (CYP) epoxygenase-dependent metabolic pathways. J. Gen. Physiol. 124, 719-727. doi: 10.1085/jgp.200409140

Williams, J. M., Sarkis, A., Hoagland, K. M., Fredrich, K., Ryan, R. P., Moreno, C., et al. (2008). Transfer of the CYP4A region of chromosome 5 from Lewis to Dahl S rats attenuates renal injury. Am. J. Physiol. Renal Physiol. 295, F1764-F1777. doi: 10.1152/ajprenal.90525.2008

Wilson, T. W., Alonso-Galicia, M., and Roman, R. J. (1998). Effects of lipidlowering agents in the Dahl salt-sensitive rat. Hypertension 31, 225-231. doi: 10.1161/01.HYP.31.1.225

Ying, C. J., Noguchi, T., Aso, H., Ikeda, K., Yamori, Y., and Nara, Y. (2008). The role of cytochrome p-450 in salt-sensitive stroke in stroke-prone spontaneously hypertensive rats. Hypertens. Res. 31, 1821-1827. doi: 10.1291/hypres.31. 1821 
Zeldin, D. C., Wei, S., Falck, J. R., Hammock, B. D., Snapper, J. R., and Capdevila, J. H. (1995). Metabolism of epoxyeicosatrienoic acids by cytosolic epoxide hydrolase: substrate structural determinants of asymmetric catalysis. Arch. Biochem. Biophys. 316, 443-451. doi: 10.1006/abbi.1995.1059

Zhao, X., Quigley, J. E., Yuan, J., Wang, M. H., Zhou, Y., and Imig, J. D. (2006). PPAR- $\alpha$ activator fenofibrate increases renal CYP-derived eicosanoid synthesis and improves endothelial dilator function in obese Zucker rats. Am. J. Physiol. Heart Circ. Physiol. 290, H2187-H2195. doi: 10.1152/ajpheart.00937.2005

Zhao, X., and Li, L. Y. (2008). PPAR- $\alpha$ agonist fenofibrate induces renal CYP enzymes and reduces blood pressure and glomerular hypertrophy in Zucker diabetic fatty rats. Am. J. Nephrol. 28, 598-606. doi: 10.1159/000116885

Zhou, Y., Luo, P., Chang, H. H., Huang, H., Yang, T., Dong, Z., et al. (2008). Colfibrate attenuates blood pressure and sodium retention in DOCA-salt hypertension. Kidney Int. 74, 1040-1048. doi: 10.1038/ki.2008.300

Zuckerman, A., Chander, P. N., Zeballos, G. A., and Stier, C. T. Jr. (1997). Regional renal nitric oxide release in stroke-prone spontaneously hypertensive rats. Hypertension 30, 1479-1486.
Conflict of Interest Statement: The authors declare that the research was conducted in the absence of any commercial or financial relationships that could be construed as a potential conflict of interest.

Received: 26 June 2014; accepted: 23 July 2014; published online: 15 August 2014. Citation: Li J, Stier CT, Chander PN, Manthati VL, Falck JR and Carroll MA (2014) Pharmacological manipulation of arachidonic acid-epoxygenase results in divergent effects on renal damage. Front. Pharmacol. 5:187. doi: 10.3389/fphar.2014.00187 This article was submitted to Experimental Pharmacology and Drug Discovery, a section of the journal Frontiers in Pharmacology.

Copyright (C) 2014 Li, Stier, Chander, Manthati, Falck and Carroll. This is an open-access article distributed under the terms of the Creative Commons Attribution License (CC BY). The use, distribution or reproduction in other forums is permitted, provided the original author(s) or licensor are credited and that the original publication in this journal is cited, in accordance with accepted academic practice. No use, distribution or reproduction is permitted which does not comply with these terms. 